Rabaska

Revue d'ethnologie de l'Amérique française

\title{
Échos du Sommet national du patrimoine bâti. Un appel à décomplexer le patrimoine
}

\section{Denis Boucher}

Volume 16, 2018

URI : https://id.erudit.org/iderudit/1051335ar

DOI : https://doi.org/10.7202/1051335ar

Aller au sommaire du numéro

Éditeur(s)

Société québécoise d'ethnologie

ISSN

1703-7433 (imprimé)

1916-7350 (numérique)

Découvrir la revue

Citer ce document

Boucher, D. (2018). Échos du Sommet national du patrimoine bâti. Un appel à décomplexer le patrimoine. Rabaska, 16, 206-211.

https://doi.org/10.7202/1051335ar d'utilisation que vous pouvez consulter en ligne. 


\title{
Colloques et rassemblements
}

\section{Échos du Sommet national du patrimoine bâti. Un appel à décomplexer le patrimoine}

\author{
DENIS BOUCHER \\ Historien et conseiller au Conseil du patrimoine culturel du Québec
}

Le $1^{\text {er }}$ novembre dernier, le Sommet national du patrimoine bâti a été une occasion de transformer un cri d'alarme en cri de ralliement pour le milieu associatif en patrimoine. Plus encore, ce rendez-vous a exprimé une volonté de décomplexer cette cause pour sortir de la posture de la sauvegarde et entrer dans celle, émergente, du projet citoyen. Retour sur la genèse de cet événement et sur quelques constats dégagés.

La tenue de ce sommet n'est pas le fruit du hasard. Pour bien en saisir l'importance et la pertinence, il convient d'élargir le regard au-delà des faits saillants de la journée pour comprendre comment cet événement visait à prendre acte des préoccupations spécifiques du milieu associatif pour jeter les bases d'un engagement renouvelé. Le regard se doit d'être aussi rétroactif afin d'entrevoir le chemin parcouru par les groupes, organismes et associations voués au patrimoine depuis 25 ans et mieux saisir les défis qui se présentent à eux pour l'avenir.

\section{Depuis le Forum québécois du patrimoine}

Il convient d'abord de souligner que l'initiative de ce Sommet, qui revient à la Table de concertation des acteurs nationaux du patrimoine bâti, rappelle les actions menées par le Forum québécois du patrimoine (FQP), actif durant les années 1990. En effet, la concertation du milieu associatif, l'identification d'attentes communes et la définition d'actions regroupées sont à la base de ces deux démarches.

En 1991, la motivation à l'origine de la création du FQP est de doter le Québec d'une politique du patrimoine. Parmi les enjeux majeurs soulevés 
alors figurentceux de la décentralisation des responsabilités et de l'implication accrue des municipalités, ce que la politique culturelle, adoptée en 1992, va d'ailleurs encourager. En effet, la multiplication des organisations locales et régionales a fragilisé les instances de portée nationale ; l'accès à des programmes d'assistance financière pour des projets à caractère national est réduit au profit d'ententes régionales et locales par où les ressources sont canalisées ${ }^{1}$. Il faut rappeler que nombre d'organisations locales et régionales tentent de consolider leur existence en assurant, tant bien que mal, la conservation de fonds d'archives ou la gestion de biens, immeubles ou sites d'intérêt patrimonial. Cette situation force ces organisations à réduire leurs actions de sauvegarde qui sont davantage menées par les instances de portée nationale. Si leurs engagements demeurent précaires et peu soutenus financièrement, leur rôle est plus concret et mieux ancré dans leurs communautés.

L'idée d'une politique du patrimoine continue d'alimenter tous les espoirs au cours des premières années du nouveau millénaire. Un projet de déclaration québécoise sur le patrimoine est diffusé en 1999 par la table de concertation du $\mathrm{FQP}^{2}$ et une proposition d'éléments pour une politique du patrimoine bâti est préparée par le Conseil des monuments et sites du Québec la même année ${ }^{3}$. Le débat s'intensifie avec le dépôt du « Rapport Arpin » pour une politique du patrimoine du Québec en 20004. L'invitation de ce groupe-conseil à mieux organiser le milieu associatif en patrimoine aura peu d'effet, même si le milieu universitaire y répond par la création de chaires de recherche, de deux instituts du patrimoine et de nombreux colloques. Certaines initiatives municipales s'en inspirent cependant pour mieux définir le rôle des organismes et musées à l'échelle locale et régionale. C'est le cas avec l'adoption des premières politiques municipales du patrimoine, notamment à Rivière-du-Loup en 2002.

En 2006, l'Observatoire de la culture et des communications publie un portrait éloquent du milieu associatif en patrimoine. On y recense pas moins de 356 organismes actifs dans ce secteur. Cependant, l'Observatoire conclut que « les organismes, associations et fédérations sont nombreux $[\ldots]$ ce qui accentue la perception d'une dispersion et d'un éclectisme de ce milieu ${ }^{5} »$.

1. Gérard Beaudet, « Une politique du patrimoine et non un patrimoine de politiques », Le Devoir, 6 janvier 1995, A9.

2. Collectif, «Le Patrimoine, une affaire de société », Le Devoir, 30 juillet 1999, A8.

3. Consulté en ligne : actionpatrimoine.ca/positions/elements-pour-une-politique-du-patrimoine-bati-au-quebec.

4. Groupe-conseil sur la Politique du patrimoine culturel du Québec, Notre patrimoine. Un présent du passé, novembre 2000, 239 p.

5. Observatoire de la culture et des communications, État des lieux du patrimoine, des institutions muséales et des archives, décembre 2006, 71 p. 
Ce portrait révèle également la grande précarité financière de la plupart de ces entités, expliquant du même coup pourquoi aussi peu de ressources professionnelles sont à leur emploi.

La même année, est amorcé le chantier de révision de la Loi sur les biens culturels, un exercice vers lequel se tournent les espoirs du milieu associatif pour une véritable vision gouvernementale en matière de patrimoine. L'entrée en vigueur de la Loi sur le patrimoine culturel, six ans plus tard, est à nouveau l'occasion d'attendre un engagement plus structuré de l'État dans la foulée de la mise en œuvre de cette loi. En 2014, le ministère de la Culture et des communications réorganise l'aide financière accordée aux organismes de portée nationale. Trois organismes dits de regroupement sont désormais reconnus et soutenus à ce titre : Archéo-Québec pour l'archéologie, le Conseil québécois du patrimoine vivant pour le patrimoine immatériel et Action patrimoine pour le patrimoine bâti et les paysages. Par conséquent, les autres organismes de portée nationale se voient retirer leur aide au fonctionnement.

\section{Enjeux actuels du milieu associatif}

Ce bref détour permet de faire ressortir trois grands constats qu'il importe d'avoir en tête pour comprendre la situation du milieu associatif en patrimoine et ainsi mieux comprendre les intentions du Sommet du patrimoine bâti en 2017.

D'abord, il convient d'admettre que, depuis 25 ans, le milieu associatif souffre d'un éclatement et d'un repli qui s'est accentué par la multiplication des nouvelles formes de patrimoine et par la dispersion des acteurs et des actions. Déjà, en 1991, le FQP avait l'intention de créer des ponts entre les instances de portée nationale et toutes les autres impliquées à des échelles locales et régionales ${ }^{6}$. Cette rupture entre ces deux échelles d'organisations apparait toujours comme un enjeu majeur. Une autre polarisation s'est accélérée autour de deux axes que sont le patrimoine matériel et le patrimoine immatériel. Plus encore, le patrimoine matériel s'est scindé en deux : l'immobilier regroupant les acteurs du patrimoine bâti et les objets, collections et archives dispersés entre les musées, fondations et autres sociétés d'histoire.

Ensuite, le milieu associatif semble avoir attendu que l'État, par le biais de grandes politiques publiques, reconnaisse le patrimoine comme un enjeu spécifique qui possède son système d'intervention propre. Ceci, afin de partager les responsabilités permettant de reconnaître un rôle clef au milieu associatif et lui accorder un soutien conséquent de l'État. Force est d'admettre

6. Gérard Beaudet, « Le Forum québécois du patrimoine, quatre ans déjà ! », Continuité, $\mathrm{n}^{\circ} 64$, printemps 1995, p. 49. 
que cette occasion ne s'est jamais présentée. Or, cette attente d'un soutien étatique n'a pas stimulé la recherche d'alliances, ni la révision des modèles d'organisation. La difficulté grandissante à fidéliser des membres cotisants a accentué la précarité financière de plusieurs organisations, les citoyens étant davantage mobilisés autour d'enjeux spécifiques ou de projets concrets.

Enfin, la gestion du patrimoine bâti s'est complexifiée avec le temps, créant un fossé entre les experts, au service des autorités publiques, et les mouvements citoyens, groupes et associations, regroupant les non-experts. De même, s'il faut saluer la professionnalisation remarquable qui s'est produite dans le champ du patrimoine, notamment par le biais de programmes de formation de pointe, celle-ci a peu profité au milieu associatif.

\section{Une Table des acteurs nationaux}

La Table a été créée en 2014 à l'initiative de l'Association des moulins du Québec (АмQ). Ce nouvel organisme, créé en 2008, souhaitait réunir les autres acteurs voués au patrimoine à l'échelle du Québec. Le succès de l'initiative est révélateur : tous conviennent de l'importance de briser l'isolement et de comprendre davantage qui fait quoi au Québec. Vingt-cinq ans après le $\mathrm{FQP}$, l'idée d'un regroupement apparaît plus pertinente que jamais ${ }^{7}$.

Les dix organismes qui adhèrent à la Table en 2015 participent à de nombreuses rencontres de réseautage et d'échange d'information ${ }^{8}$. Surtout, la Table permet de faire apparaître un état des lieux du milieu associatif, révélant beaucoup de ressemblances dans la réalité de chaque organisation, mais, aussi et surtout, l'envie de travailler ensemble.

Les rencontres de la Table ont aussi pour résultat de mieux comprendre la situation du milieu associatif. Limites de l'action de sauvegarde in extremis, essoufflement des organisations, éclatement des forces, manque d'appui des autorités publiques, manque de relève, financement en baisse ; le diagnostic est accablant. Néanmoins, les idées et la volonté d'agir demeurent riches et nourrissent un exercice important mené par la Table : préparer un mémoire commun dans la foulée des consultations en vue du renouvellement de la politique culturelle du Québec. Des séances de travail permettent d'atteindre de nombreux consensus sur les propositions à soumettre au ministre de

7. Au sujet de la Table de concertation des acteurs nationaux du patrimoine bâti, on peut consulter le dossier spécial publié sur le sujet dans le magazine Continuité, n ${ }^{\circ} 155$, hiver 2018.

8. Les organismes qui composent la Table de concertation des acteurs nationaux du patrimoine bâti en date de mai 2018 sont : l'Association des moulins du Québec, Action patrimoine, l'Héritage canadien du Québec, Docomomo Québec, l'Association québécoise pour le patrimoine industriel, l'Association des interprètes du patrimoine, le Conseil du patrimoine religieux du Québec, la Fédération Histoire Québec, l'Association des amis et propriétaires de maisons anciennes du Québec et la Corporation des gestionnaires de phares de l'estuaire et du golfe Saint-Laurent. 
la Culture et des communications d'alors. La rédaction de ce mémoire représente un premier test pour la Table et donne le goût de partager à plus grande échelle les propositions du mémoire à l'occasion d'un événement visant à élargir le débat, impliquer d'autres acteurs et faire entendre plus fortement la voix de la Table. L'idée d'organiser un sommet national du patrimoine bâti fait rapidement l'unanimité.

\section{Quelques constats dégagés du Sommet national du patrimoine bâti}

Soulignons d'embléel'exploit que représente l'organisation de cet événement d'envergure par une table de concertation sans permanence et sans moyens, dans des délais très courts et face à des attentes énormes ${ }^{9}$. Le Sommet avait pour but d'éclairer les tendances actuelles en matière d'implication citoyenne et d'actions de sauvegarde afin de mieux situer le rôle du milieu associatif. Il s'agissait aussi d'exposer les principaux obstacles au développement de ces organisations et de proposer des solutions. Aussi, chaque organisation devait exposer ses propres préoccupations afin alimenter les débats qui seraient menés dans trois ateliers aux thématiques croisées ${ }^{10}$.

Le grand succès de participation - forçant les organisateurs à refuser de nombreux participants en raison de la capacité des salles limitée à 150 personnes - a révélé l'intérêt du milieu à se réunir davantage. De plus, il convient de souligner l'importante présence de nouveaux acteurs et représentants de la relève. Cette présence de nouveaux visages et l'énergie qui s'en est dégagée a contribué à donner le ton à cette journée pour en faire une occasion de motivation et d'inspiration.

Mais plus encore, le Sommet a permis de dégager quelques constats importants, qu'il serait opportun de résumer par l'idée de décomplexer le patrimoine ${ }^{11}$. D'abord, tout au long de la journée, il a été question d'initiatives citoyennes pour valoriser des formes nouvelles de patrimoine qui n'attendent plus les reconnaissances officielles et les procédures formalisées pour agir.

9. Il importe de saluer ici l'efficacité de l'équipe d'Action patrimoine qui a pu assurer la logistique de la journée et le dévouement du petit comité organisateur qui, au nom de la Table, a tenté de répondre de son mieux aux multiples et légitimes attentes des organisations.

10. Pour une synthèse complète du Sommet, on peut consulter les Échos du Sommet du patrimoine bâti en ligne : sommetpatrimoinebati.ca/wp-content/uploads/2018/04/Echos_Sommet-patrimoinebati-2018.pdf.

11. J'avais utilisé le terme décomplexé lors d'une conférence prononcée au Sommet et portant sur les enjeux actuels pour qualifier certaines tendances émergentes qui amènent le milieu associatif à jouer un rôle accru dans l'organisation d'actions citoyennes. Essentiellement, mon propos visait à esquisser les changements importants qui apparaissent dans la façon d'apprécier, de défendre et d'expliquer le patrimoine. Une vision plus communautaire se répand aujourd'hui dans un contexte de démocratisation des héritages culturels, d'émergence de nouvelles communautés d'appartenance et de revendications grandissantes des citoyens pour s'approprier leur territoire. J'invitais les défenseurs du patrimoine à se défaire de certains complexes associés à cette cause et à épauler les initiatives d'une relève qui entend faire sa place. 
Actuellement, les mouvements en faveur du patrimoine ont recours à une multitude de plateformes virtuelles et de réseaux d'information et d'échange. Cette situation accorde une place accrue aux savoirs profanes, amateurs, bénévoles et érudits. Ces citoyens, mobilisés et réseautés par l'entremise de ces outils de communication efficaces, accèdent à une quantité sans précédent d'information. Tout ceci permet aux citoyens de prendre davantage la parole. Dans ce contexte, le milieu associatif est appelé à jouer un rôle accru pour organiser ces mouvements et guider l'action.

D'autre part, nombre d'intervenants souhaitent sortir de l'image des vieilles pierres pour s'allier au mouvement en faveur d'un aménagement plus durable du territoire. Au confluent d'enjeux urbanistiques, culturels, sociaux, communautaires et démocratiques, le patrimoine est au cœur du mouvement des défenseurs du territoire, sans pour autant en être partie prenante. Cette intention de décloisonner le patrimoine pour rejoindre les revendications territoriales des citoyens marque une volonté de s'allier au discours alternatif qui a su fédérer nombre d'enjeux liés à la qualité des milieux de vie.

Enfin, plusieurs discussions ont valorisé l'idée du patrimoine-projet. Moins portés à défendre un patrimoine d'exception, marqué par une architecture exceptionnelle ou par sa participation à la Grande Histoire, les mouvements de sauvegarde s'activent davantage autour de projets communautaires. La conservation du patrimoine se justifie moins par elle-même qu'à travers la préservation d'un usage ou le développement de nouvelles fonctions utiles à la communauté. Ceci provoque un intérêt pour de nouvelles formes de patrimoines, parfois plus récents, souvent plus modestes, mais surtout revendiqués comme autant de lieux d'ancrage de nouvelles communautés d'appartenance.

En guise de conclusion, il convient d'admettre que le milieu associatif, malgré son éclatement, est plus que jamais le moteur de l'action patrimoniale. Après des années de revendications répétées en faveur d'un cadre spécifique d'intervention déterminé par les autorités, le mouvement patrimonial est peut-être en train de se refonder sur ses bases citoyennes et communautaires. Dans cet esprit, le regroupement des actions locales, régionales et nationales s'avère essentiel, pour peu qu'on puisse faire tomber les cloisons et les complexes, et ainsi mieux organiser les forces vives du patrimoine du Québec. 